\title{
The bZIP protein from Tamarix hispida, ThbZIP1, is ACGT elements binding factor that enhances abiotic stress signaling in transgenic Arabidopsis
}

\author{
Xiaoyu Ji, Guifeng Liu, Yujia Liu, Lei Zheng, Xianguang Nie and Yucheng Wang*
}

\begin{abstract}
Background: Tamarix spp. are woody halophyte, which are very tolerant to abiotic stresses such as salinity and drought, but little is known about their specific stress response systems. Basic leucine zipper proteins (bZIPs) play important roles in the ability of plants to withstand adverse environmental conditions. However, their exact roles in abiotic stress tolerance are still not fully known. In the current study, we functionally characterized a bZIP gene (ThbZIP1) from Tamarix hispida in response to abiotic stresses.

Results: We addressed the regulatory network of ThbZIP1 in three levels, i.e. its upstream regulators, the cis-acting elements recognized by ThbZIP1, and its downstream target genes. Two MYCs were found to bind to E-box, in the promoter of ThbZIP1 to activate its expression. Expression of ThbZIP1 is induced by ABA, salt, drought, methyl viologen and cold. ThbZIP1 can specifically bind to ACGT elements, with the highest binding affinity to the C-box, followed by the G-box and lastly the A-box. Compared with wild-type (Col-0) Arabidopsis, transgenic plants expressing ThbZIP1 had an increased tolerance to drought and salt, but had an increased sensitivity to ABA during seed germination and root growth; meanwhile, ROS level, cell death and water loss rate in transgenic plants were significantly reduced. Microarray analyses showed that many ROS scavenging genes were up-regulated by ThbZIPI under salt stress conditions.
\end{abstract}

Conclusions: Based on these data, we suggest that ThbZIP1 confers abiotic stress tolerance through activating stress tolerance genes to modulate ROS scavenging ability and other physiological changes involved in stress tolerance, and plays an important role in the ABA-mediated stress response of T. hispida.

Keywords: Abiotic stress, bZIP transcription factor, Arabidopsis thaliana, Gene expression regulation, Tamarix hispida, Yeast one-hybrid

\section{Background}

Basic leucine zipper proteins (bZIPs) are a large family of transcription factors (TFs) in plants that contain a characteristic and highly conserved basic domain with two structural features: a basic domain responsible for sequence-specific DNA binding and an adjacent heptad leucine repeat domain referred to as a leucine zipper dimerization motif [1,2]. The bZIPs are involved in diverse physiological processes such as seed maturation and germination, plant senescence [2], photomorphogenesis and light signaling [3], and also play important

\footnotetext{
* Correspondence: ychngwang@yahoo.com

State Key Laboratory of Tree Genetics and Breeding (Northeast Forestry University), 26 Hexing Road, 150040 Harbin, China
}

roles in the ability of plants to withstand adverse environmental conditions. Transformed plants that overexpress $b Z I P$ genes have improved tolerance to stresses of salt $[4,5]$, water deficits [6,7], freezing [1,8], methyl viologenoxidative [1], heat shock [9], hypersensitivity to ABA [10], and pathogen infections [2]. In addition, the bZIP gene is also found to reprogram amino acid metabolism during low energy stress [11].

The $b Z I P$ genes can regulate the expression of genes involved in stress tolerance. For example, a $b Z I P$ gene from Poncirus trifoliata can upregulate the genes involved in stress tolerance, including $L E A, C D P K$ and DREB [6]. Overexpression of a tomato $b Z I P$ gene can regulate stressrelated genes, such as AtRD29A, AtCOR47 and the SlCI7like dehydrin [12]. The Arabidopsis bZIP28 can form a 
transcriptional complex that upregulates the expression of ER stress-induced genes [13]. The investigation of the target genes regulated by bZIPs on a genome scale may provide information regarding the gene expression regulation network mediated by this TF. Some studies have investigated the target genes regulated by bZIPs, but only a few target genes were investigated, and the study of target genes regulated by bZIP on a genome-wide scale is needed.

Tamarix hispida Willd. is a woody halophyte that can grow well in drought prone soils and salinity soil. Previously, we had cloned a bZIP gene (ThbZIP1) from T. hispida, and the transgenic tobacco overexpression of ThbZIP1 showed improved salt tolerance [14]. In the current study, we further showed that the ThbZIP1 gene is transcriptionally regulated by $M Y C$ genes. ThbZIP1 specifically binds to ACGT elements, including C-box, G-box and A-box, and can upregulate serial stress related genes. Moreover, overexpression of ThbZIP1 led to a reduction in the cellular levels of ROS, cell death and water loss rate under salt, drought and ABA treatment conditions. These results suggest that ThbZIP1 plays a pivotal role in the fine-tuning ABA signaling and controls ROS accumulation.

\section{Results}

\section{Cloning the promoter of ThbZIP1 and analysis of its} activity

The cloned promoter of ThbZIP1 is 1,571 bp in length. To analyze promoter activity of the promoter of ThbZIP1, Arabidopsis plants harboring the ProThbZIP1:: GUS transgene were analyzed using GUS staining (Additional file 1: Figure S1A). The results showed that GUS activity can be detected in all tissues of Arabidopsis, including seeds, pistils, anthers and stamens, in addition to whole seedlings at different developmental stages (Additional file 1: Figure S1B), which indicates that it has high promoter activity in different plant tissues.

\section{The expression of ThbZIP1 is activated by ThMYC6}

The cis-elements in the promoter of ThbZIP1 were predicted, and diverse cis-elements were found, such as E-box, ABRE, DOFCOREZM, MYBCORE and W-box (Additional file 2: Figure S2). Additionally there were seven E-box ("CANNTG") motifs in this promoter (Additional file 2: Figure S2), indicating that E-box motif is important for regulating the expression of ThbZIP1. Therefore, yeast one-hybrid analysis was performed to investigate the TFs that can bind to E-box motif. Two $M Y C$ genes (ThMYC4 and ThMYC6) were found to bind to the E-box motif (Figure 1B). In addition, ThMYC6 bound more strongly to E-box than ThMYC4, which was used for further study. To further understand the specificity of these interactions, the E-box motif was mutated (Figure 1A) and the interaction between the TFs and the mutated motifs were investigated using yeast onehybrid analysis. The results showed that ThMYC6 failed to interact with these mutated motifs (Figure 1B), which indicates the specificity of its binding to E-box motif.

To further determine if ThMYC6 can activate the expression of ThbZIP1 by binding to E-box motifs in the promoter, the pHIS2 vectors that contained the ThbZIP1 promoter fragments with E-box motifs (pHIS2-ProE $(+)$ ) and without E-box motif (pHIS2-ProE(-)) (Figure 1C) interacted with the pGADT7 constructs that harboring ThMYC6. The results indicated that ThMYC6 can interact with the promoter fragment containing the E-box motifs, but failed to interact with the promoter fragment that lacked the E-box motifs (Figure 1D).

To further confirm the interaction of ThMYC6 with E-box, the effector construct (pROKII-ThMYC6) was co-transformed into tobacco leaves together with its corresponding reporter plasmids (pROKII-ThMYC6 cotransformed with pCAM-E-box, pCAM-E-M3, pCAME-boxp + or pCAM-E-boxp-, respectively). Histochemical staining and a GUS activity assay showed that the GUS gene was activated in the tobacco cells when the cotransformation of pROKII-ThMYC6 with pCAM-E-box or pCAM-E-boxp + were performed. However, the cotransformation of pROKII-ThMYC6 with pCAM-E-boxp-, and the negative controls all failed in GUS activation (Figure 1F, G). These data clearly indicated that ThMYC6 can activate gene expression through its interaction with the ThbZIP1 promoter via binding to the E-box motifs.

\section{The expression of ThbZIP1 is induced by abiotic stresses and $A B A$}

Previously, we had studied the expression of ThMYC6, and the results showed that it can be highly induced by salt, osmotic and ABA treatments [15]. In the present study, real-time RT-PCR results showed that ThbZIP1 is highly induced by treatments with $\mathrm{NaCl}$, PEG6000, ABA, methyl viologen (MV) or cold (Figure 2). These results suggested that both ThMYC6 and ThbZIP1 respond to abiotic stresses and are involved in the ABA signaling pathway.

\section{The motif sequences recognized by ThbZIP1}

The ThbZIP1 can bind to C-, G- and A-box sequences, but binds more strongly to C-box, followed by G-box and lastly A-box motifs (Figure 3A). Among the four mutants of ACGT elements, ThbZIP1 fails to bind to the CM2 mutant, but is able to bind to the CM1, CM3 and CM4 to some extent. Furthermore, ThbZIP1 is able to bind to CM3 more strongly than CM1 and CM4 (Figure 3A). To confirm these interactions, the effector construct (pROKII-ThbZIP1) and reporter plasmids (pCAM-C-box, -G-box or -A-box) were coexpressed in 
A

\section{E-box:CAAATG \\ E-M1: $\underline{\text { ACAATG }}$ \\ E-M2:CAAACA \\ E-M3: $\underline{\text { ACCGCA }}$}

B

(a)

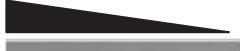

(b)

(c)

(d)

(e)

(f)

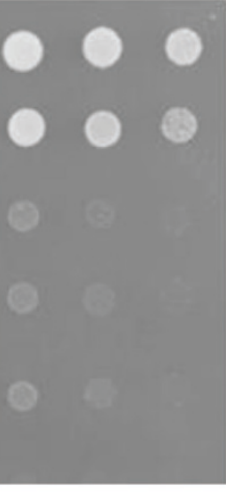

(ThMYC6)

E

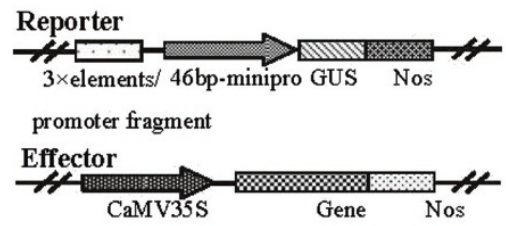

F
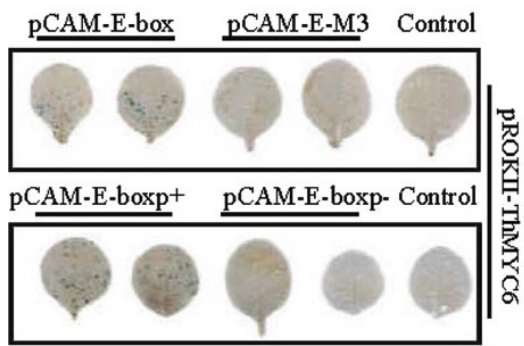

C

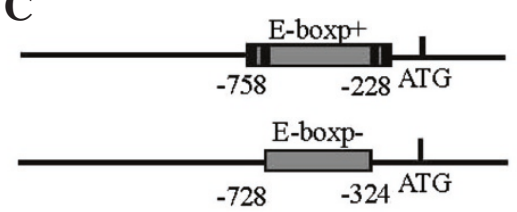

D

(a)

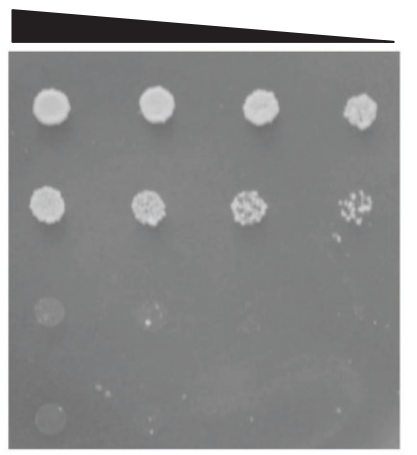

(ThMYC6)

\section{G}

Reporter Effector

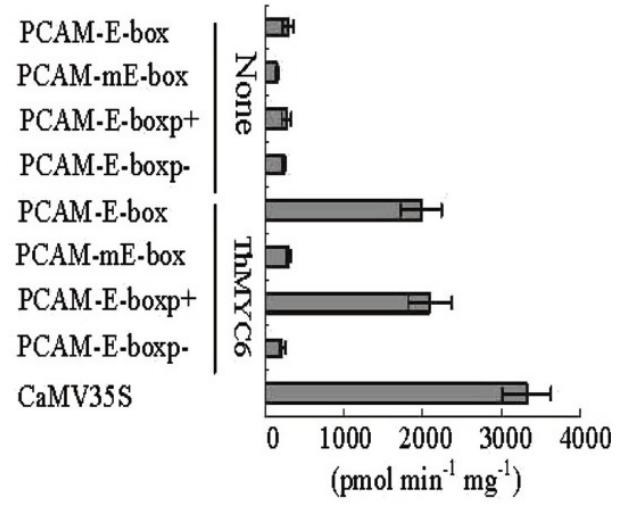

Figure 1 Analyses of the upstream regulator of ThbZIP1. A: E-box and its mutated sequences. B: The bindings of ThMYC6 / ThMYC4 to E-box and the mutated E-box sequences. a: pHIS2-p53 + pGAD-Rec2-p53 (positive control); b: pHIS2-E-box + pGAD-Rec2-ThMYC6/ThMYC4; c: pHIS2-E-M1 + pGAD-Rec2-ThMYC6/ThMYC4; d: pHIS2-E-M2 + pGAD-Rec2-ThMYC6/ThMYC4; e: pHIS2-E-M3 + pGAD-Rec2-ThMYC6/ThMYC4; f: pHIS2-p53 + pGAD-Rec2ThMYC6 /ThMYC4 (negative control). pGAD-Rec2-53: p53 as a fusion with the GAL4 AD in PGAD-Rec. pHIS2-E-box, -E-M1, -E-M2, -E-M3: three tandem copies of E-box, its mutants E-M1, E-M2 and E-M3 (shown in Figure 1A) respectively inserted into pHIS2. pGAD-Rec2-ThMYC4, -ThMYC6: ThMYC4 and ThMYC6 as a fusion with the GAL4 AD in PGADT7-Rec2. The triangle indicates serial dilutions (1/1, 1/10, 1/100). C: The promoter fragments of ThbZIP1 with or without the cis-elements used in analyses of the yeast one-hybrid and coexpression of reporter and effector vectors. The black lines indicate the E-box, and the grey lines show the promoter fragments. D: The bindings of TFs to promoter fragments with or without the E-box motifs using yeast one-hybrid analysis (shown in Figure 1C). a: pHIS2- p53 + pGADT7-Rec2-p53(positive control); b: pHIS2-ProE(+) + pGADT7-Rec2-ThMYC6/ThMYC4; c: pHIS2-ProE(-) + pGADT7-Rec2-ThMYC6/ThMYC4; d: pHIS2-p53 + pGADT7-Rec2-ThMYC6/ThMYC4 (negative control). pHIS2-ProE(+), -ProE(-): pHIS2 harboring the promoter fragments containing E-box, or without E-box motifs. E: Diagrams of the reporter and effector vectors. F: The coexpression of reporter and effector vectors in tobacco leaves. PCAM-E-box, -E-M3: three tandem copies of E-box, or its mutant E-M3 were respectively fused to a minimal promoter to drive GUS. -E-boxp+, -E-boxp: one copy of promoter fragment containing E-box, or without E-box was respectively fused to a minimal promoter for driving GUS. pROKII-ThMYC6: the ORF of ThMYC6 was under the control of CaMV 355 promoter. G: The GUS activity assay of the coexpression of reporter and effector plasmid. Error bars indicate SE.

the tobacco leaves. Consistent with the yeast one-hybrid analyses, GUS staining and activity measurements both showed that ThbZIP1 can bind more strongly to C-box sequences, followed by G-box and A-box (Figure 3C, D).

\section{Constitutive expression of ThbZIP1 enhances salt} tolerance

Nine independent $T_{3}$ homozygous lines overexpressing ThbZIP1 were generated, and RT-PCR confirmed that 


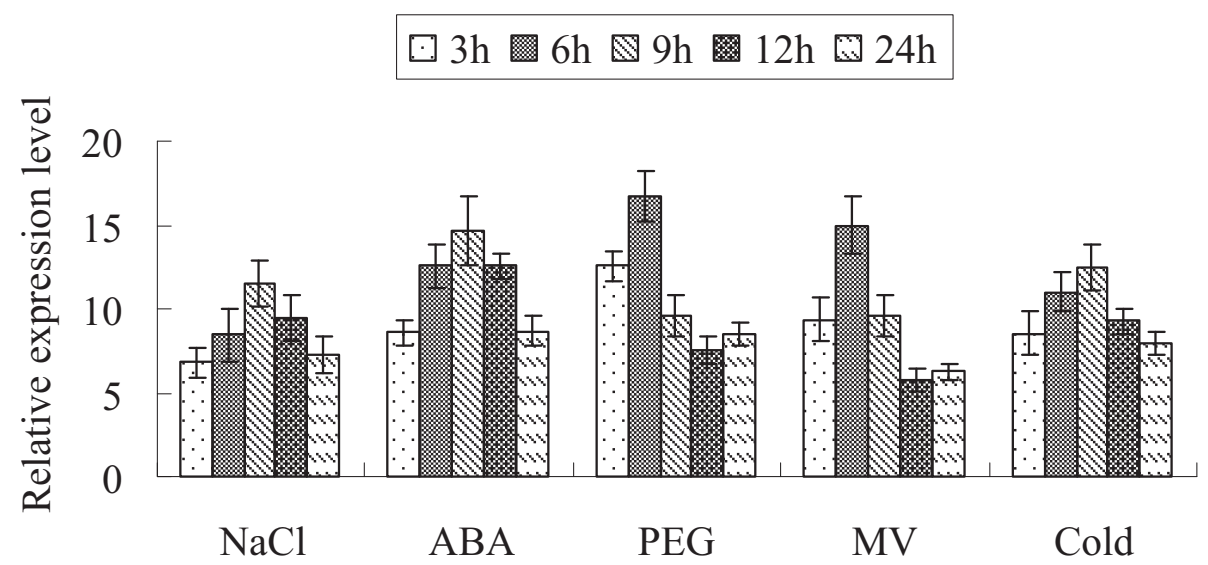

Figure 2 The expression patterns of ThbZIP1 in response to abiotic stresses and ABA. The expression patterns of ThbZIP1 in response to salt, osmotic, oxidative, and cold stress and ABA treatment. The error bars were obtained from multiple replicates of the real-time PCR.

the exogenous ThbZIP1 was expressed in the transgenic plants (Figure 4A). Two independent ThbZIP1 transgenic lines (b-2, b-8) were selected for further study. Under normal growth conditions, there was no difference in growth between Col-0 and transgenic plants (Figure 4B, C, D). However, the transgenic lines showed significant improved root growth and fresh weight under drought or salt stress as compared with wild-type (Col-0) plants. In addition, the ThbZIP1 transgenic plants exhibited an increase in seed germination under $\mathrm{NaCl}$ or Mannitol stress in comparison with Col-0 plants (Figure 4C, D). These results suggest that ThbZIP1 conferred an enhanced salt and drought tolerance to ThbZIP1 transformed plants.

In the absence of exogenously applied ABA, the percentage of successful germinations of transgenic seeds and plant growth were similar to those of Col-0 seeds. However, the germination of ThbZIP1 transgenic seeds was more sensitive to exogenously applied ABA as compared to Col-0 (Figure 4C, D). There were no obvious differences in the above ground organs between the ThbZIP1-transformed plants and Col-0, but the root length of the ThbZIP1 transgenic plants was shorter in response to exogenously applied ABA (Figure 4B, D).

\section{The target genes regulated by ThbZIP1}

The expression profile changes between Col-0 and ThbZIP1 overexpressed plants were compared under normal growth and salt stress conditions using Agilent Arabidopsis Oligo microarray. A total of 241 and 322 genes were upregulated and downregulated in ThbZIP1 overexpressed plants, respectively, under normal growth condition (Additional file 3: Table S1). Under salt stress conditions, 1,204 and 1,228 genes were significantly upregulated and downregulated in ThbZIP1-transformed plants, respectively (Additional file 4: Table S2).
Twenty-four significantly differentially expressed genes (12 genes in normal condition, and 12 genes in the $\mathrm{NaCl}$ response) identified by microarray were randomly selected for real-time RT-PCR analyses. There were high correlation coefficients between the real-time PCR and microarray data $\left(\mathrm{R}^{2}=0.9817, \mathrm{P}<0.05\right.$ under normal growth conditions; $\mathrm{R}^{2}=0.9764, \mathrm{P}<0.05$ under salt stress conditions) (Additional file 5: Figure S3), which validates the reliability of the microarray results.

Gene Ontology (GO) analysis was conducted of the pathways significantly $(\mathrm{P}<0.05)$ enriched in the differentially regulated genes (Additional file 6: Table S3). Under salt stress conditions, the upregulated genes were significantly enriched in 17 subgroups, and the downregulated genes were significantly enriched in 16 subgroups. Among these enriched subgroups, seven subgroups were enriched for both up- and down-regulated genes, such as binding, metabolic process, cellular process and response to stimulus, indicating that these pathways were highly altered in response to salt stress. The subgroup of catalytic activity pathways were only enriched by the upregulated genes under salt stress, indicating that this pathway may play a positive role in ThbZIP1-modulated salt stress response.

To study whether the genes can be directly regulated by ThbZIP1 via binding to the C-, G- or A-box in their promoters, we randomly selected the genes upregulated by ThbZIP1 and screened their promoter regions for searching C-, G- or A-box motifs. The results showed that all of these genes have at least one C-box, G-box or A-box and most of them have two or more C-, G- or A-box motifs in their promoter regions (Additional file 7: Table S4). This result suggested that ThbZIP1 can directly regulate the expression of genes via binding to the C-, G- or A-box motifs present in their promoters. 
A
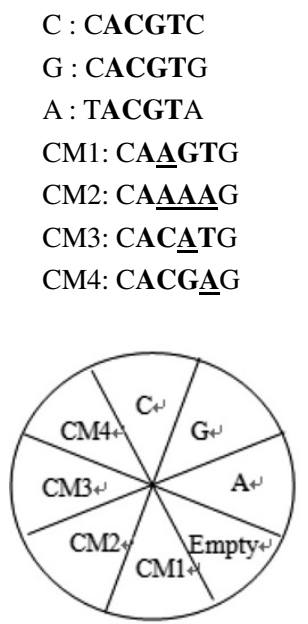

B

\section{Reporter}
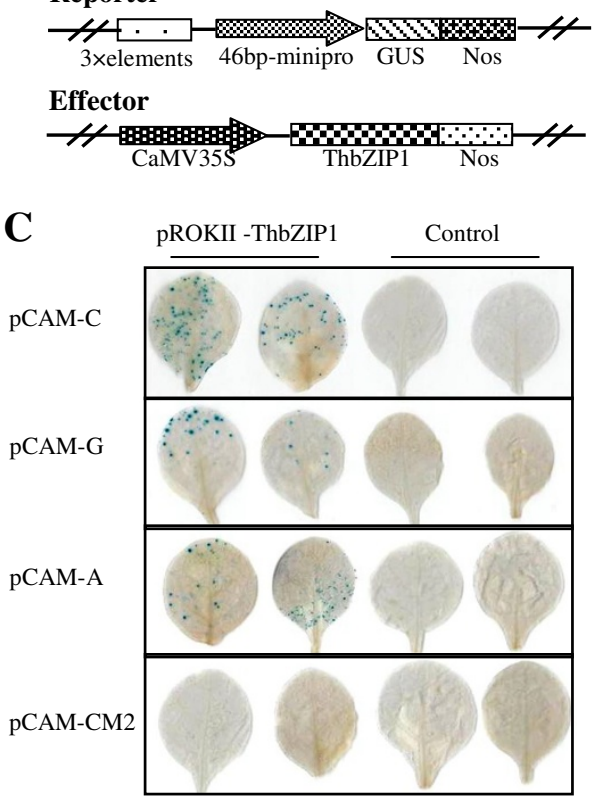
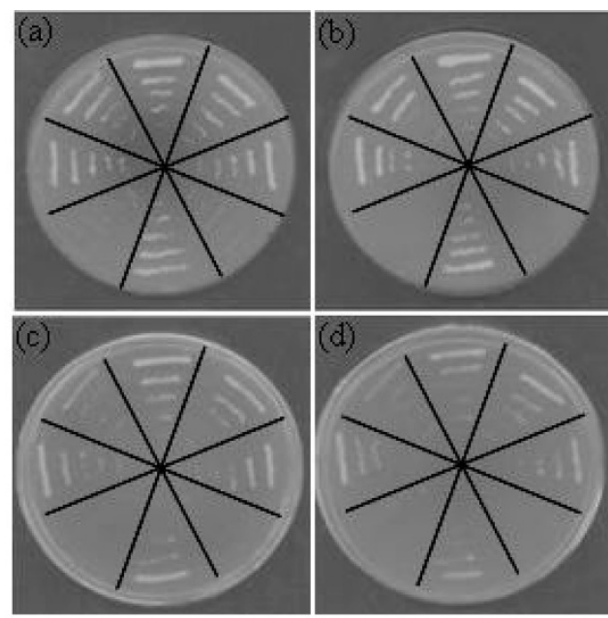

D

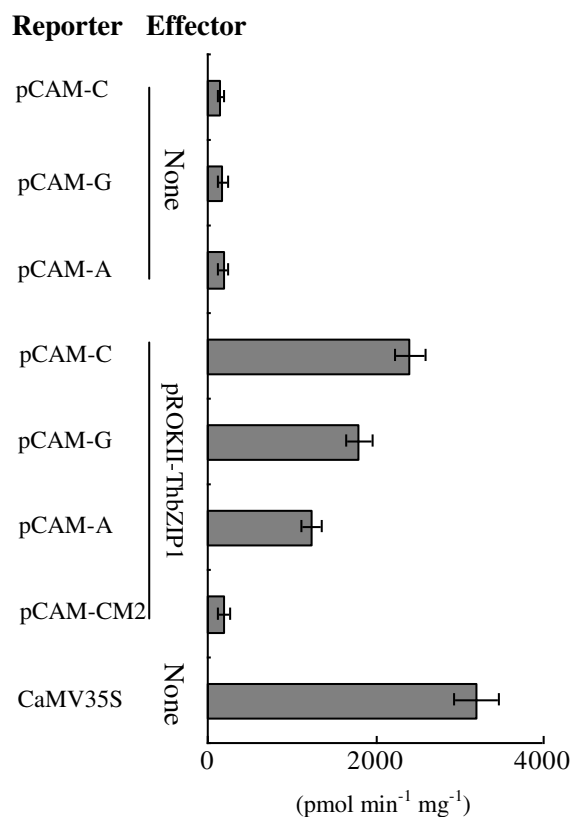

Figure 3 Analyses of the bindings of ThbZIP1 to C-, G- and A-box. A: (a-d): Analysis of the bindings of ThbZIP1 to C-, G-, A-box and their mutated sequences by yeast one-hybrid analysis. The yeast cells were grown on different intensities of selective dropout medias: SD/- Trp-Leu/-His (TDO) + 3-AT (3-AT concentrations, a: 30 mM, b: 40 mM, c: 50 mM, d: 60 mM). B: A diagram of the reporter and effector vectors. C: The coexpression of reporter and effector vector in tobacco leaves. PCAM-C, -G -A, -CM2: three tandem of C-, G-, A-box, or their mutant CM2 was respectively fused to a minimal promoter $(-46$ to +1$)$ to drive GUS. pROKII-ThbZIP1: the ORF of ThbZIP1 was under the control of CaMV 355 promoter. D: GUS activity assay of the coexpression of reporter and effector plasmid. CaMV35S: The transformation of pCAMBIA1301 alone (positive control). The transformation of the reporter plasmids alone were used as negative controls. All assays were repeated three times and error bars indicate SE.

\section{Physiological roles mediated by of ThbZIP1}

The cellular levels of $\mathrm{O}^{2-}$ and $\mathrm{H}_{2} \mathrm{O}_{2}$, the two prominent ROS species, were compared between transgenic and Col-0 plants by NBT and DAB in situ staining, respectively. The steady state levels of both $\mathrm{H}_{2} \mathrm{O}_{2}$ and $\mathrm{O}^{2-}$ were displayed as deep brown and dark blue products, respectively, and were highly reduced in leaves of ThbZIP1 transgenic plants compared with Col-0 plants under salt, drought and $\mathrm{ABA}$ stress conditions (Figure $5 \mathrm{~A}, \mathrm{~B})$. Consistent with $\mathrm{DAB}$ staining, $\mathrm{H}_{2} \mathrm{O}_{2}$ content measurement also showed that there was no difference in $\mathrm{H}_{2} \mathrm{O}_{2}$ content between transgenic and Col-0 plants under normal growth condition, but $\mathrm{H}_{2} \mathrm{O}_{2}$ content in the transgenic plants was significantly decreased compared with that in Col-0 plants under salt and ABA treatment (Figure 5E). 


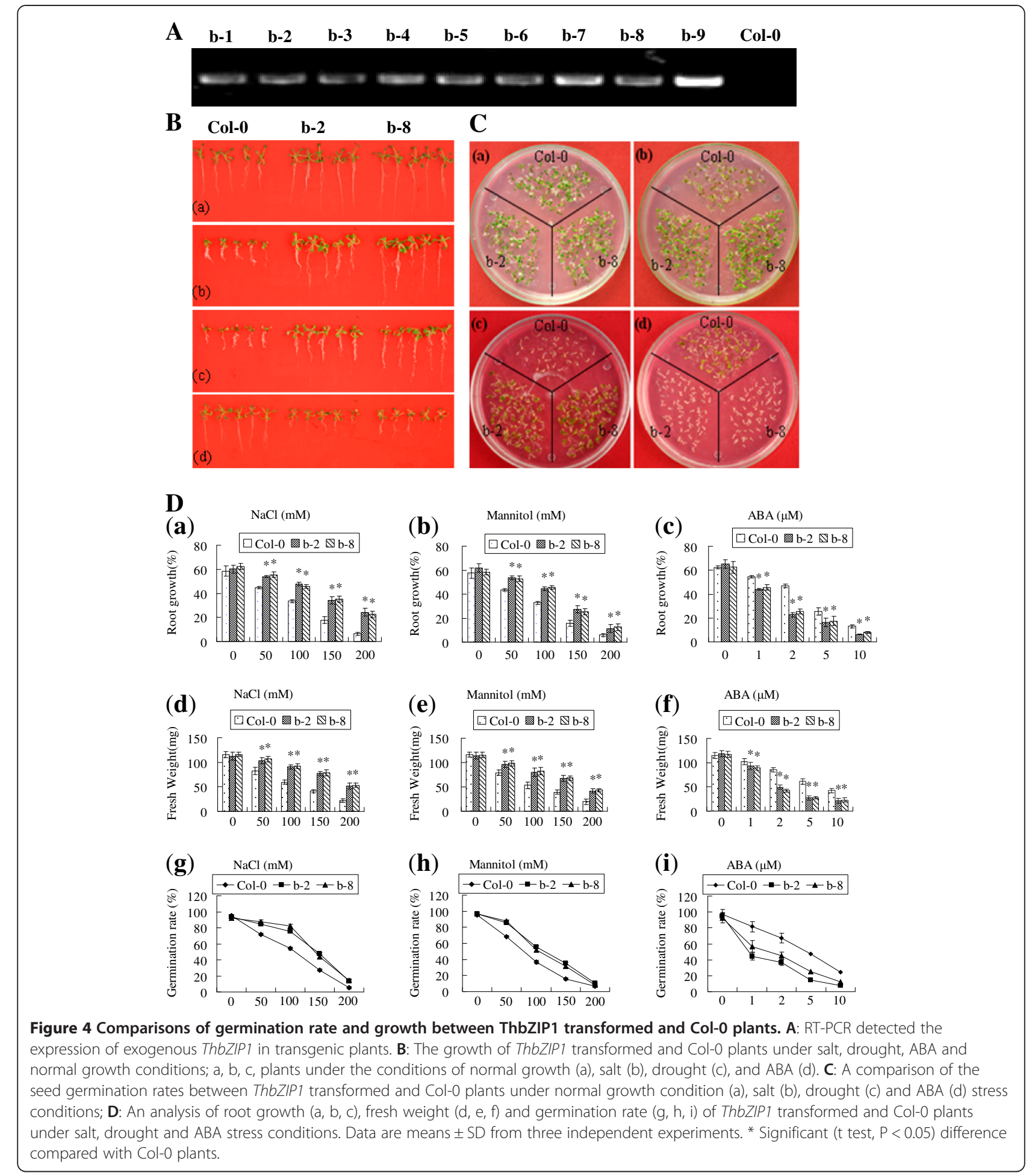

The cellular level of ROS in guard cells and root tips were examined using $\mathrm{H}_{2}$ DCF-DA fluorescence staining. ROS levels in the guard cells and the root tips of the transgenic plants were notably lower than those in Col-0 plants (Figure 5C, D). These observations indicated that ThbZIP1 expression enables plants cells to decrease ROS amounts to alleviate the damage caused by stress.

Under salt stress conditions, 7 unique GST genes were highly upregulated by overexpression of ThbZIP1, and no GSTs were downregulated (Additional file 4: Table S2); therefore, we further studied the GST 


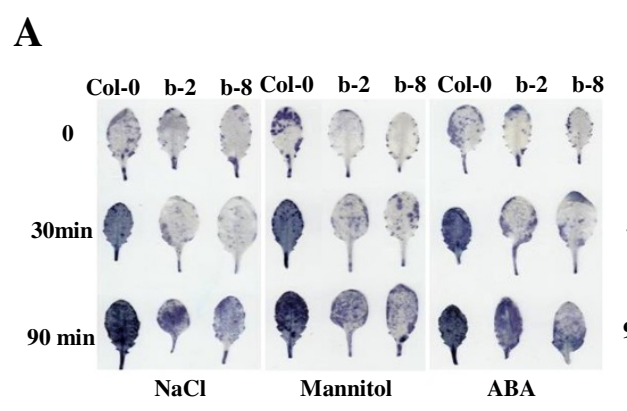

C
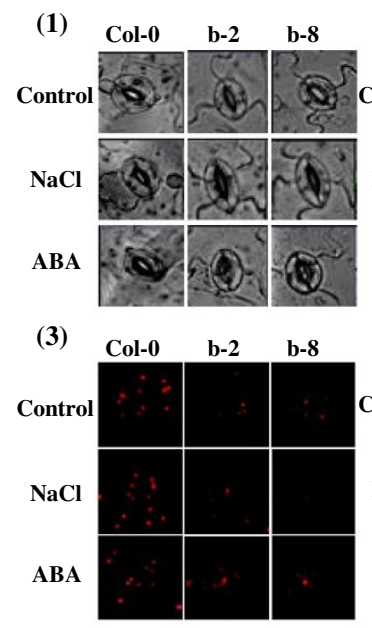

(2)

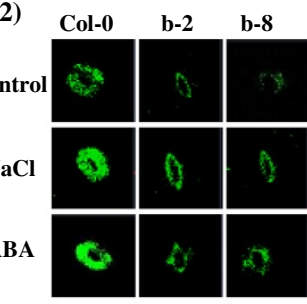

(4) Col-0 $\quad$ b-2 $\quad$ b-8

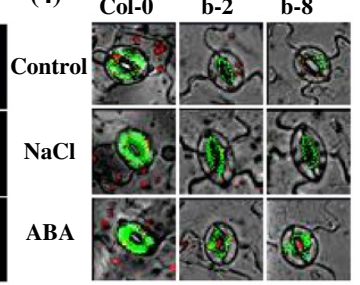

\section{B}

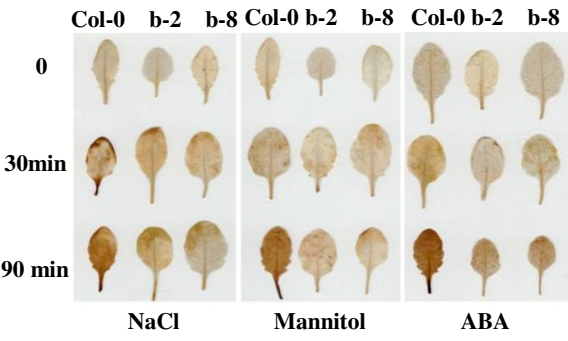

D

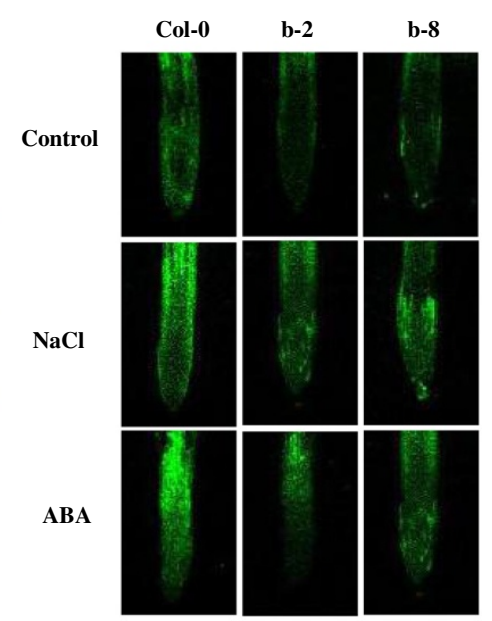

$\mathbf{E}$

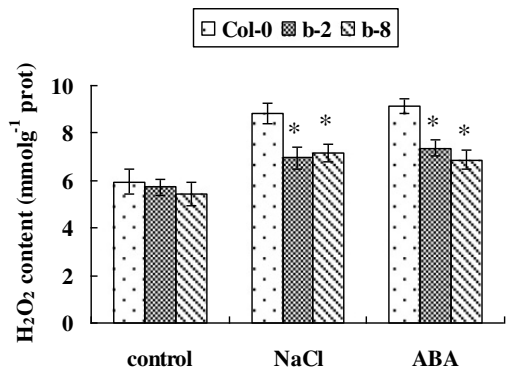

$\mathbf{F}$

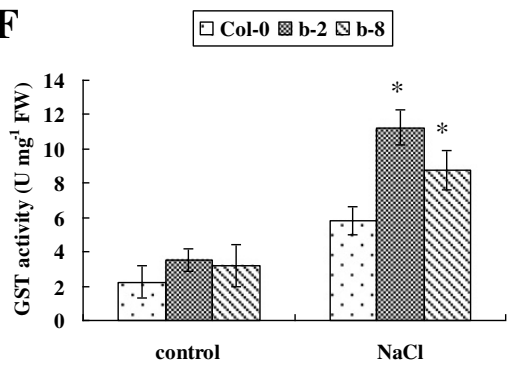

Figure 5 The constitutive expression of ThbZIP1 decreases ROS levels and enhanced GST activity. A, B: The leaves from Col- 0 and transgenic plants were pretreated with $\mathrm{NaCl}$, mannitol, or $\mathrm{ABA}$, and were stained with $\mathrm{NBT}$ to visualize $\mathrm{O}^{2-}(\mathbf{A})$, or were stained with $\mathrm{DAB}$ to visualize $\mathrm{H}_{2} \mathrm{O}_{2}$ (B). C: Analyses of ROS production in intact guard cells of transgenic and Col-0 plants indicated by $\mathrm{H}_{2} \mathrm{DCF}-\mathrm{DA}$. Epidermal peels were loaded with $\mathrm{H}_{2} \mathrm{DCF}-\mathrm{DA}$ after the incubation with $10 \mu \mathrm{M}$ ABA or $150 \mathrm{mM} \mathrm{NaCl}$ for $2 \mathrm{~h}$. (1) peel cells imaged under bright field; (2) ROS in guard cells were detected by $\mathrm{H}_{2} \mathrm{DCF}-\mathrm{DA}$ (ROS shown as green fluorescence); (3) Chloroplast in the leaves that shown as red fluorescence; (4) merge of bright field and fluorescence (chloroplast shown as red fluorescence). D: Detection of ROS accumulation in root tips of transgenic and Col-0 plants using $\mathrm{H}_{2} \mathrm{DCF}-\mathrm{DA}$ staining method. E: Measurement of $\mathrm{H}_{2} \mathrm{O}_{2}$ level in transgenic and Col-0 plants. F: Comparison of GST activity between Col-0 and ThbZIP1-transformed plants. All experiments were repeated three times. Data are means \pm SD from three independent experiments. * Significant ( $t$ test, $P<0.05)$ difference compared with Col-0 plants.

activities in response to salt stress. Consistent with the gene expression profiles of GSTs, the transgenic and Col-0 plants showed similar GST activities prior to stress, but the transgenic lines showed significantly higher GST activities than Col-0 plants did under salt stress conditions (Figure 5F). Taken together, these results suggested that GST genes play important roles in
ThbZIP1 mediated elimination of cellular ROS generated by salt stress.

Evans blue staining showed that the cell death in ThbZIP1 transgenic plants were notably reduced after salt, drought and ABA treatments compared with the Col-0 plants (Figure 6A). The levels of cell death in root tips were examined using propidium iodide (PI) fluorescence 


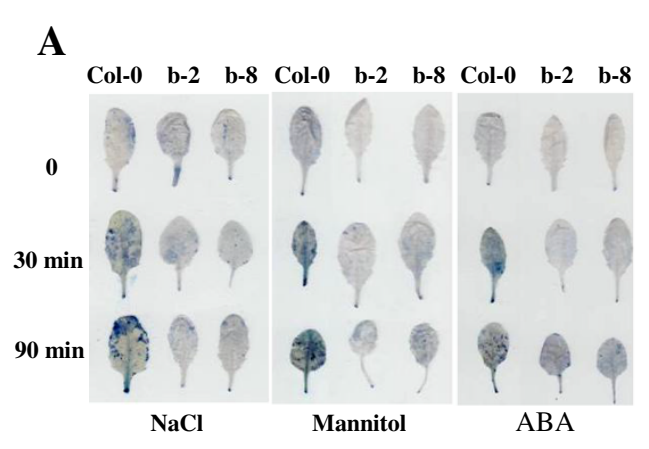

\title{
B
}

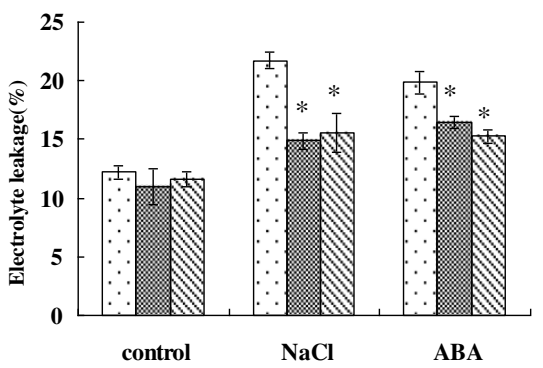

C

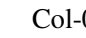

\begin{abstract}
b-2
\end{abstract}
b-8

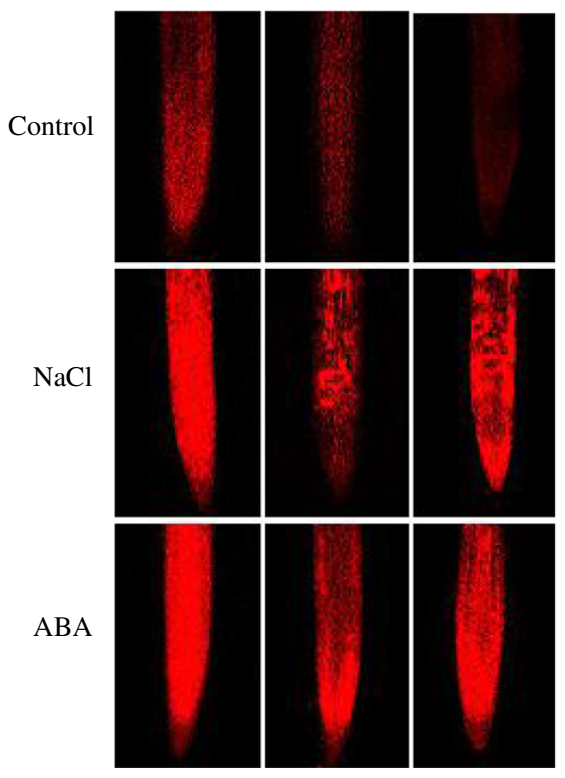

D

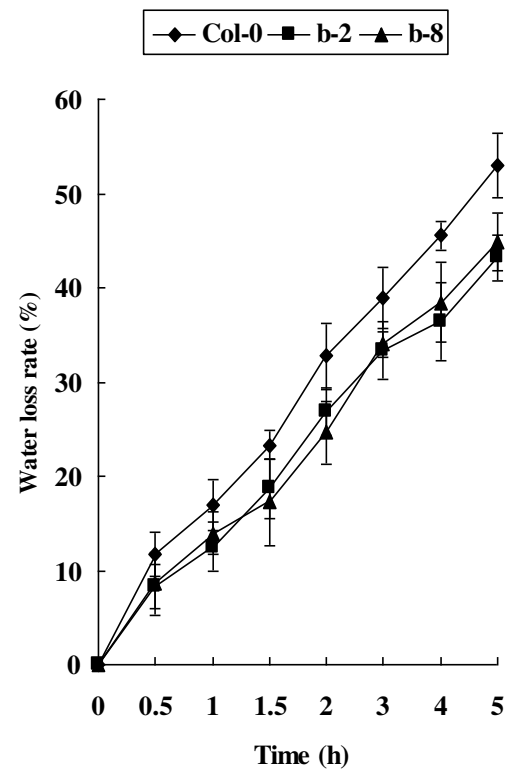

Figure 6 The constitutive expression of ThbZIP1 decreases cell death and water loss rate under stress conditions. A: Cell death staining with Evans blue before (Control) and after the treatment of $\mathrm{NaCl}$, mannitol, or ABA. B: Comparison of electrolyte leakage between Col-0 and ThbZIP1 transformed plants. C: Assay of cell death between transgenic and Col-0 plants using propidium iodide (PI) fluorescence staining. D: Transpirational water loss rate analyses in Col-0 and ThbZIP1 transformed plants. All experiments were repeated three times. Data are means \pm SD from three independent experiments. * Significant (t test, $\mathrm{P}<0.05$ ) difference compared with Col-0 plants.

staining. As shown in Figure 6C, under normal, ABA and salt stress conditions, there were notably fewer dead cells in the transgenic plants than in Col-0 plants. Moreover, electrolyte leakage assay also showed that after salt and ABA treatments electrolyte leakage in transgenic lines was significantly lower than in Col-0 plants (Figure 6B). This result was consistent with the results of Evans blue and PI staining, which indicated that the overexpression of ThbZIP1 could protect the cells from death under stress conditions.

In addition, the water loss rate assay showed that the leaves of ThbZIP1-expressing transgenic plants exhibited delayed water loss rate relative to Col-0 plant leaves under dehydration conditions (Figure 6D), which demonstrated that the capacity to conserve water is enhanced by the expression of ThbZIP1.

\section{Discussion}

Despite the fact that transformation of $b Z I P$ genes leads to improved abiotic stress tolerance, the molecular mechanisms of the tolerance and sensitivity remain largely unknown and were explored here. The microarray results showed that compared under normal growth condition, the number of differentially expressed genes increased by nearly 5 -fold under salt stress conditions (Additional file 4: Table S2). This indicated that the expression of this TF alone is not sufficient to activate many stress-related genes, but needs to be combined with stress signals. Previous studies also supported this point; for example, a bZIP protein, SIAREB, alone cannot activate the expression of AtRD29A in Arabidopsis until ABA is supplemented [12]. Also, the expression of succinate 
dehydrogenase $2-3$ and $L E A$ can only be regulated by ABI3 in Arabidopsis in the presence of ABA [16].

Modulation of ROS levels is critical for the tolerance of abiotic stresses, given that ROS levels can reflect the damage of cellular components and act as signaling molecules at low concentrations [9]. Plants possess a complex antioxidant system to detoxify stress-induced ROS, which includes several enzymes to scavenge ROS and protect the cells against oxidative stress. The microarray results showed that compared with in Col- 0 plants, the ROS scavenging genes in transgenic plants found in higher levels included GST, POD, SOD, L-ascorbate peroxidase $(A P X)$, thylakoidal ascorbate peroxidase and glutathione peroxidase (Additional file 4: Table S2). GSTs are involved in plant cell stress signaling and provide protection by detoxifying endogenous plant toxins under stress conditions [17]. This report has demonstrated that; (1) constitutive expression of ThbZIP1 leads to 7 unique GSTs that were highly upregulated under salt stress conditions, and no GSTs were downregulated (Additional file 4: Table S2); correspondingly, GST activity in transgenic plants was highly improved (Figure 5E); (2) ROS levels were greatly decreased in ThbZIP1 overexpressed plants compared with Col-0 under salt stress conditions (Figure 5A-D); (3) overexpression of ThbZIP1 conferred tolerance to salt stresses (Figure 4); (4) GSTs can efficiently detoxify ROS induced in plants by various stress stimulus. These results combined strongly indicate that GSTs play an important role in ThbZIP1 mediated ROS scavenging to enhance plant tolerance. In addition, our previous studies showed that ThbZIP1 can improve the activities of POD and SOD, and decreased MDA levels under salt stress conditions [14]. These results together suggested that ROS scavenging capacity is enhanced in the plants that overexpress ThbZIP1 under stress conditions. This conclusion was also further supported by the histochemical assay described here. DAB and NBT staining, $\mathrm{H}_{2} \mathrm{O}_{2}$ measurement and ROS detection in guard cells and root tips using $\mathrm{H}_{2}$ DCF-DA all clearly demonstrated that the ThbZIP1 transgenic lines accumulated remarkably less ROS compared with Col-0 under stress conditions (Figure 5A-E). Furthermore, Evens blue and PI staining and electrolyte leakage assay all showed a decrease in cell death rates in ThbZIP1 transgenic plants under stress conditions compared with Col-0 plants (Figure 6A-C). These combined results indicate conclusively that ThbZIP1 plays a critical role in ROS scavenging, which prevents the oxidative damage of cells and decreases cell death.

What deserves to be mentioned is that, although ThbZIP1-transformed Arabidopsis plants showed improved ROS scavenging and decreased membrane damage under ABA treatment, still they are sensitive to ABA. Obviously, ABA may cause damage to the plants overexpressing ThbZIP1 through other pathways rather than ROS injury and membrane damage.

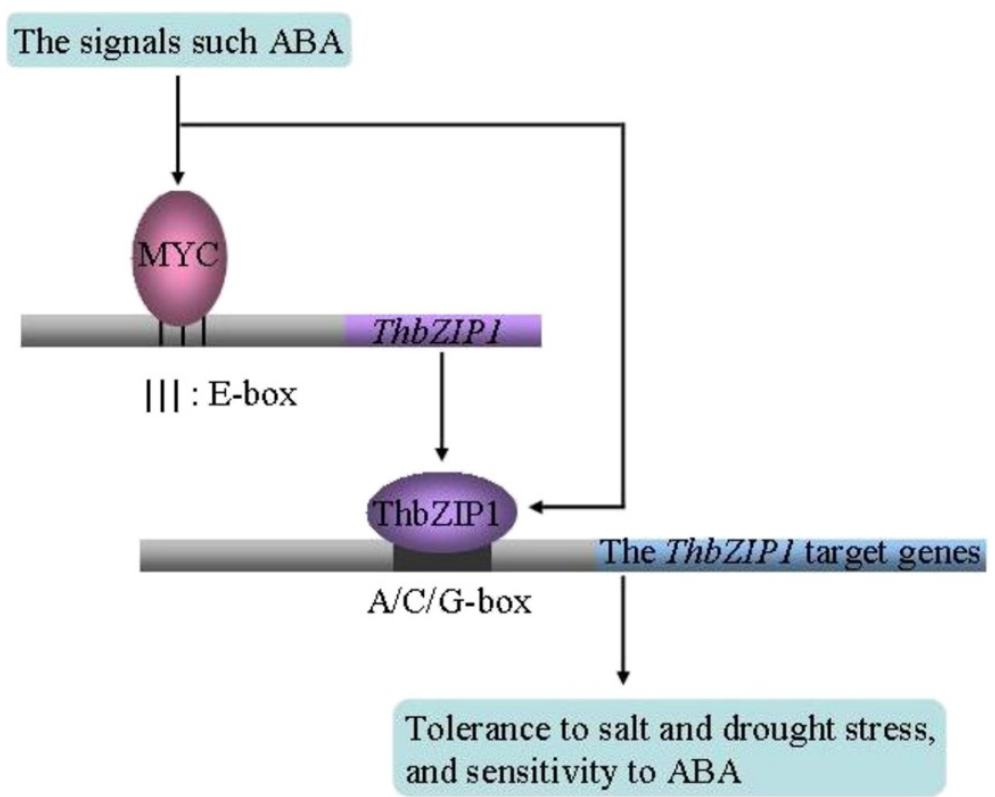

Figure 7 Model of the regulatory network of ThbZIP1 involved in abiotic stress responses. Stress signals such as ABA are produced in plants when exposed to abiotic stressors, and the expression of MYC is induced by stress signals; then the MYC proteins bind to the E-box motif in the promoter of ThbZIP1 to activate the expression of ThbZIP1. The ThbZIP1 in turn binds to the A-, C- or G-box in the promoter of the genes to alter their expression, resulting that abiotic stress tolerances are improved in plants. 


\section{Conclusion}

Based on all the experimental data presented here, a model is proposed to explain the ThbZIP1-mediated salt tolerance (Figure 7). Stress signals such as ABA are generated during abiotic stresses, which activate the expression of the transcription factors including MYC; then, the MYC proteins bind to E-box motifs in the ThbZIP1 promoter to induce its expression. Combined with the stress signals, the activated ThbZIP1 in turn binds to the A-, Cor G-box motifs in the promoters of genes to activate their expression, leading to the improvement of abiotic stress tolerance in plants. Therefore, ThbZIP1 is a factor in modulating abiotic stress responses through an ABAdependent signaling pathway.

\section{Methods}

\section{Plant materials and growth conditions}

Seedlings of $T$. hispida were grown in a greenhouse under controlled conditions of $70 \%$ relative humidity, light/dark cycles of $14 / 10 \mathrm{~h}$, and were maintained at $24^{\circ} \mathrm{C}$. To induce abiotic stresses, the seedlings were at $4^{\circ} \mathrm{C}$ or watered on their roots with a solution of $0.4 \mathrm{M} \mathrm{NaCl}, 20 \%(\mathrm{w} / \mathrm{v})$ PEG6000, $100 \mu \mathrm{M}$ ABA or $50 \mu \mathrm{M}$ MV for 3, 6, 9, 12 and $24 \mathrm{~h}$; the seedlings watered with water were harvested at the corresponding time points as controls.

\section{Plant transformation}

The ORF of ThbZIP1 was inserted into pROKII driven by CaMV35S promoter, designated as pROKII-ThbZIP1 (see Additional file 8: Table S5 for primers used). The pROKII-ThbZIP1 was transformed into Arabidopsis (ecotype Columbia) using the floral dip method.

\section{Cloning and activity analysis of the ThbZIP1 promoter}

Based on the sequence of ThbZIP1 (GenBank number: FJ752700), the promoter of ThbZIP1 (1,571 bp in length) was cloned using a Genome Walking Kit (Takara, Dalian, China). The potential cis-regulatory elements in the promoter were predicted using the software PLACE [18]. The $35 \mathrm{~S}$ promoter in pCAMBIA1301 was replaced with the ThbZIP1 promoter that fused with the 5' UTR of ThbZIP1 to drive the $\beta$-glucuronidase (GUS) gene (ProThbZIP1:: GUS; Additional file 1: Figure S1A). The ProThbZIP1:: GUS construct was transferred into Arabidopsis plants by the floral dip method. The $\mathrm{T}_{3}$ seedlings were used for promoter activity analysis.

\section{Analysis of the upstream regulators of ThbZIP1}

Previously, eight transcriptomes from roots of T. hispida treated with $\mathrm{NaHCO} 3$ for $0,12,24$ and 48 h (two biological replicates were set at each time point) were built [19]. In total, 47,324 unigenes were generated after these transcriptomes de novo assembly using SOAPdenovo. The TFs from different families were identified, PCR amplified and cloned into pGADT7-Rec2 (Clontech, Palo Alto, CA, USA) to form a cDNA library (designed as TFs library) for yeast one-hybrid assay. There are seven E-box motifs ("CANNTG") in the promoter of ThbZIP1. Three tandem copies of E-box motif were cloned into a pHIS2 vector (designed as pHIS2-E-box; see Additional file 8: Table S6 for primers used), and were screened with a TFs library for yeast one-hybrid assay (Clontech, Palo Alto, CA, USA). The interactions of pHIS2-p53 (three tandem copies of the cis-acting DNA consensus sequence in pHIS2, which is recognized by p53) with the tested TFs were used as negative controls.

Two MYC (ThMYC4 and ThMYC6 GenBank number: JN166788 and JN166790) were identified to bind to the E-box motif, of which ThMYC6 that bound with greater strength to the E-box was used for further study. The E-box core motif of "CANNTG" was mutated to "ACAATG", "CAAACA", and "ACCGCA" (designed as pHIS2-E-M1, -E-M2, -E-M3, respectively; see Additional file 8: Table S6 for primers used). The interactions of ThMYC6 with E-box motif and its corresponding mutated motifs were studied using the yeast one-hybrid analysis.

To determine if ThMYC6 is able to activate the expression of ThbZIP1 by interacting with the E-box motifs, the pHIS2 constructs that harbored the promoter fragments of ThbZIP1 which contained E-boxes (pHIS2-ProE(+)), or without E-boxes (pHIS2-ProE(-)) were respectively generated as reporter vectors (see Additional file 8: Table S6 for primers used). The interactions of these constructs with ThMYC6 were studied using yeast one-hybrid analysis.

To further verify these interactions, the three tandem copies of the E-box and its mutant E-M3 (ACCGCA) were respectively fused to the minimal $35 \mathrm{~S}$ promoter $(-46$ to +1$)$ to drive GUS, and designed as pCAME-box and pCAM-E-M3 (see Additional file 8: Table S7 for primers used). The promoter fragments of ThbZIP1, which contained E-box motifs (named as pCAM-E-boxp+), and lacked E-box motifs (pCAM-E-boxp-) (Figure 1C) were respectively fused to the minimal $35 \mathrm{~S}$ promoter to drive GUS as reporter vectors (see Additional file 8: Table S7 for primers used). The effector vector was constructed by cloning the full ORF of ThMYC6 into pROKII driven by the $35 \mathrm{~S}$ promoter (named as pROKIIThMYC6). Both of the reporter vectors and their corresponding effector vectors were co-transformed into tobacco leaves using the particle bombardment. The transformation of pCAMBIA1301 alone (CaMV35S) was used as positive control. The transformation of the reporter plasmids alone or effector plasmids alone was used as negative controls. All assays were repeated three times. GUS histochemical staining assay was performed as described by Jefferson [20], and the GUS stained leaves were scanned by using scanner (D4800,UNISPLENDOUR, China). GUS activity levels were determined according to Jefferson [21]. 


\section{Real-time PCR analysis of gene expression}

The real-time RT-PCR was performed using $\alpha$-tubulin (XM_002301092) and actin 3 (XM_002308329) as internal controls (see Additional file 8: Table S8 for primers used). PCR was performed on a MJ Research OpticonTM ${ }^{2}$ instrument with the following conditions: $94^{\circ} \mathrm{C}$ for $30 \mathrm{~s}, 45$ cycles of $94^{\circ} \mathrm{C}$ for $12 \mathrm{~s}, 58^{\circ} \mathrm{C}$ for $30 \mathrm{~s}$, $72^{\circ} \mathrm{C}$ for $40 \mathrm{~s}$, and $80^{\circ} \mathrm{C}$ for $1 \mathrm{~s}$ for a plate reading. The relative expression levels of the products were calculated according to the $2^{-\Delta \Delta \mathrm{Ct}}$ method [22]. Relative gene expression levels were calculated as the transcription level under stress treatment divided by the transcription level of the controls (i.e., samples from plants grown under normal conditions and harvested at the same treatment time points).

\section{Assay of ThbZIP1 bindings to C-box, G-box and A-box motifs}

Three tandem copies of C-box, G-box and A-box, together with their mutants, CM1: CAAGTG, CM2: CAAAAG, CM3: CACATG and CM4: CACGAG, were cloned into a pHIS2 vector (see Additional file 8: Table S6 for primers used), respectively. Yeast one-hybrid screening analysis was performed to study their interactions with ThbZIP1. The yeast cells were grown on selective dropout media: SD/- Trp-Leu/-His (TDO) + 3-AT (3-AT concentration from 30 to $60 \mathrm{mM}$ ). Three tandem copies of the C-box, G-box and A-box and the mutant sequence CM2 (CAAAAG) were fused to the minimal $35 \mathrm{~S}$ promoter $(-46$ to +1$)$ for driving GUS (constructs containing C-box, G-box, A-box and mutant sequence CM2 named as pCAM-C, pCAM-G, pCAM-A, and pCAM-CM2, respectively). The effector vector was constructed by cloning the ORF of ThbZIP1 into pROKII driven by the $35 \mathrm{~S}$ promoter (pROKII-ThbZIP1) (see Additional file 8: Table S7 for primers used). Both the reporter and effector vectors were co-transformed into tobacco leaves using particle bombardment. GUS staining and GUS activity assay were determined as above.

\section{Analysis of $A B A$, salt and drought stress tolerances}

The $\mathrm{T}_{3}$ generation of ThbZIP1 transgenic plants were used in ABA, salt and drought stress tolerance tests. The seeds were sown on MS medium for three days and germinated seeds were transferred into a $1 / 2 \mathrm{MS}$ medium plus $0,1,2,5$, and $10 \mu \mathrm{M} \mathrm{ABA}$; or $0,50,100,150$ and $200 \mathrm{mM} \mathrm{NaCl}$; or 0, 50, 100, 150 and $200 \mathrm{mM}$ Mannitol for two weeks, respectively. The root length and fresh weight were measured. The seeds were sown on a $1 / 2$ MS medium plus $0,1,2,5$, or $10 \mu \mathrm{M} \mathrm{ABA}$, or 0,50 , 100,150 or $200 \mathrm{mM} \mathrm{NaCl}$, or $0,50,100,150$ or $200 \mathrm{mM}$ mannitol for one week, and the germination rates of each transgenic plant or Col-0 plant were measured.

\section{Microarray experiments and data analysis}

The four-week-old seedlings of Col-0 and ThbZIP1 transgenic plants without treatment or subjected to $150 \mathrm{mM} \mathrm{NaCl}$ for $3 \mathrm{~h}$ were used for the microarray analyses and three independent biological replications were performed. The Agilent Arabidopsis Oligo microarrays were employed. A Welch's t-test was used for the parametric test, and the Benjamini and Hochberg false discovery rate for multiple testing corrections was used with a P-value of $<0.05$ to filter and identify reliable genes. All genes that were considered to show significant expression level differences by these tests were then filtered by a fold change $>2.0$. For verification of microarray results, 24 differentially expressed genes identified by microarray were randomly selected for real-time RTPCR analyses.

\section{Searching for ThbZIP1-binding sequences in gene promoters}

The genes up-regulated by ThbZIP1 under normal growth or salt stress conditions were randomly selected for ThbZIP1-binding sequences in their promoter regions. The promoter sequences (from -1 to -900 ) of these genes were derived from TAIR database (http://www.arabidopsis. org/). For identification of ThbZIP1-binding motifs, the sequences of C-, G- and A-box were searched in the promoter regions of these genes.

\section{Detection of ROS and cell death}

Arabidopsis leaves from the two transgenic lines and Col-0 subjected to the ABA, $\mathrm{NaCl}$ or Mannitol treatments were infiltrated with 3, 30-diaminobenzidine (DAB) solutions or nitroblue tetrazolium (NBT) following the procedures described by Zhang et al. [23]. Cell death was examined by Evans blue staining as described by Kim et al. [24]. ROS production in intact guard cells and root tips were detected using 2, 7-dichlorofluorescin diacetate $\left(\mathrm{H}_{2} \mathrm{DCF}-\mathrm{DA}\right)$ as described by Pei et al. [25]. $\mathrm{H}_{2} \mathrm{O}_{2}$ levels and GST activity were measured according to Thordal-Christensen et al. [26] and Terada et al. [27]. Five-day-old seedlings were transferred into MS medium or MS medium with $125 \mathrm{mM} \mathrm{NaCl}, 10 \mu \mathrm{M} \mathrm{ABA}$ and placed vertically. After stress for $24 \mathrm{~h}$, at least 9 seedlings of each line were incubated with $1 \mathrm{mg} / \mathrm{mL}$ PI (Invitrogen) for $20 \mathrm{~min}$. The root tips of stained seedlings were visualized by LSM710 microscope (Zeiss, Jena, Germany) with excitation at $488 \mathrm{~nm}$ and emission at $516 \mathrm{~nm}$, respectively.

\section{Measurement of electrolyte leakage and water loss rates}

Electrolyte leakage was measured according to Liu et al. [13]. For water loss rates measurements, leaves were detached and weighed immediately (fresh weight, FW), and were then left on the laboratory bench (humidity, $45-50 \%, 20-22^{\circ} \mathrm{C}$ ) and weighed at designated time 
intervals (desiccated weights). Leaves were finally ovendried for $24 \mathrm{~h}$ at $80^{\circ} \mathrm{C}$ to a constant dry weight (DW). Water contents (WC) were measured according to the formula: $\mathrm{WC}(\%)=($ desiccated weight $-\mathrm{DW}) /(\mathrm{FW}-$ DW) $\times 100$.

\section{Statistical analyses}

Statistical analyses were carried out using SPSS 16.0 (SPSSInc, Chicago, III, USA) software. Data were compared using Student's t-test. Differences were considered to be significant if $\mathrm{P}<0.05$. ** represented $0.001<\mathrm{P}<0.01$ and ${ }^{*}$ represented $0.01<\mathrm{P}<0.05$.

\section{Additional files}

Additional file 1: Figure S1. Promoter activity assay of the ThbZIP1 promoter. A: Schematic map of the ThbZIP1 promoter inserted into pCAMBIA1301 vector. B: Test of the ThbZIP1 promoter activity in Arabidopsis plants. Seeds (a), three-day-old seedlings (b), five-day-old seedlings (c), one-week-old seedlings (d), ten-day-old seedlings (e), leaf $(f)$, root (g), flower (h), stamen (i), pistil (j, k).

Additional file 2: Figure S2. The promoter sequence of ThbZIP1 and analysis of the important cis-elements within the promoter region. The cis-elements predicted by PLACE software are shown in different colors.

Additional file 3: Table $\mathbf{S 1}$. The significantly differentially regulated genes $(p<0.05$; ratio $>2$ or $<0.5)$ in ThbZIP1 transformed plants relative to Col-0 under normal growth condition.

Additional file 4: Table S2. The significantly differentially regulated genes $(p<0.05$; ratio $>2$ or $<0.5)$ in ThbZIP1 transformed plants relative to Col-0 under salt stress conditions.

Additional file 5: Figure S3. Comparison of the results of microarray and real-time RT-PCR. The significantly differentially regulated genes detected by microarray were randomly selected for real-time RT-PCR analysis. Correlation analysis of the results between real-time RT-PCR and cDNA microarray were calculated $(P<0.05)$.

Additional file 6: Table S3. Go analysis of the genes in response to $\mathrm{NaCl}$ treatment.

Additional file 7: Table S4. The significantly upregulated genes in transgenic plants overexpression of ThbZIP1.

Additional file 8: Primers used in the study. Table S5. Primer sequences used in construction of the overexpression vectors of ThbZIP1 and ThbZIP1 fused with GFP. Table S6. The primer sequences used in the yeast one-hybrid analyses. Table S7. The primers used for construction of the reporter vectors. Table S8. The primer sequences used in real-time RT-PCR

\section{Authors' contributions}

YW and $G L$ conceived the project and designed experiments. $X J, Y L, L Z$, and $X N$ participated in the experiments. $X J$ performed data analysis. $X J$, and $Y C$ Wang drafted the manuscript. YW provided reagents and analysis tools. All authors read and approved the final manuscript.

\section{Acknowledgements}

This work was supported by The National Natural Science Foundation of China (No. 30972386), and Foundation for the Author of National Excellent Doctoral Dissertation of China (200973).

Received: 24 June 2013 Accepted: 1 October 2013

Published: 4 October 2013

\section{References}

1. Vinson CR, Sigler PB, MCKnight SL: Scissors-grip model for DNA recognition by a family of leucine zipper proteins. Science 1989, 246:911-916.

2. Lee SC, Choi HW, Hwang IS, Choi du S, Hwang BK: Functional roles of the pepper pathogen-induced bZIP transcription factor, CAbZIP1, in enhanced resistance to pathogen infection and environmental stresses. Planta 2006, 224:1209-1225.

3. Mallappa C, Yadav V, Negi P, Chattopadhyay S: A basic leucine zipper transcription factor, G-box-binding factor 1, regulates blue light-mediated photomorphogenic growth in Arabidopsis. J Biol Chem 2006, 281:22190-22199.

4. Orellana S, Yañez M, Espinoza A, Verdugo I, González E, Ruiz-Lara S, Zou M Guan Y, Ren H, Zhang F, Chen F: A bZIP transcription factor, OsABI5, is involved in rice fertility and stress tolerance. Plant Mol Biol 2008, 66:675-683.

5. Amir Hossain M, Lee Y, Cho Jl, Ahn CH, Lee SK, Jeon JS, Kang H, Lee CH, An $G$, Park PB: The bZIP transcription factor OSABF1 is an ABA responsive element binding factor that enhances abiotic stress signaling in rice. Plant Mol Biol 2010, 72:557-566

6. Huang XS, Liu JH, Chen XJ: Overexpression of PtrABF gene, a bZIP transcription factor isolated from Poncirus trifoliata, enhances dehydration and drought tolerance in tobacco via scavenging ROS and modulating expression of stress-responsive genes. BMC Plant Biol 2010, 10:230.

7. Kim JS, Mizoi J, Yoshida T, Fujita Y, Nakajima J, Ohori T, Todaka D, Nakashima K, Hirayama T, Shinozaki K, Yamaguchi-Shinozaki K: An ABRE promoter sequence is involved in osmotic stress-responsive expression of the DREB2A gene, which encodes a transcription factor regulating drought-inducible genes in Arabidopsis. Plant Cell Physiol 2011, $52: 2136-2146$

8. Liao Y, Zou HF, Wei W, Hao YJ, Tian AG, Huang J, Liu YF, Zhang JS, Chen SY: Soybean GmbZIP44, GmbZIP62 and GmbZIP78 genes function as negative regulator of $A B A$ signaling and confer salt and freezing tolerance in transgenic Arabidopsis. Planta 2008, 228:225-240.

9. Zhang X, Wollenweber B, Jiang D, Liu F, Zhao J: Water deficits and heat shock effects on photosynthesis of a transgenic Arabidopsis thaliana constitutively expressing ABP9, a bZIP transcription factor. J Exp Bot 2008, 59:839-848.

10. Lu G, Gao C, Zheng X, Han B: Identification of OsbZIP72 as a positive regulator of $A B A$ response and drought tolerance in rice. Planta 2009, 229:605-615.

11. Dietrich $\mathrm{K}$, Weltmeier F, Ehlert A, Weiste C, Stahl M, Harter K Dröge-Laser W: Heterodimers of the Arabidopsis transcription factors $b Z I P 1$ and $b Z I P 53$ reprogram amino acid metabolism during low energy stress. Plant Cell 2011, 23:381-395.

12. Hsieh TH, Li CW, Su RC, Cheng CP, Sanjaya, Tsai YC, Chan MT: A tomato bZIP transcription factor, SIAREB, is involved in water deficit and salt stress response. Planta 2010, 231:1459-1473.

13. Liu JX, Howell SH: bZIP28 And NF-Y transcription factors Are activated by ER stress and assemble into a transcriptional complex to regulate stress response genes in Arabidopsis. Plant Cell 2010, 22:782-796.

14. Wang Y, Gao C, Liang Y, Wang C, Yang C, Liu G: A novel bZIP gene from Tamarix hispida mediates physiological responses to salt stress in tobacco plants. J Plant Physiol 2010, 167:222-230.

15. Ji X, Wang Y, Liu G: Expression analysis of MYC genes from Tamarix hispida in response to different abiotic stresses. Int J Mol Sci 2012, 13:1300-1313.

16. Roschzttardtz H, Fuentes I, Vásquez M, Corvalán C, León G, Gómez I, Araya A, Holuigue L, Vicente-Carbajosa J, Jordana X: A nuclear gene encoding the iron-sulfur subunit of mitochondrial complex II is regulated by B3 domain transcription factors during seed development in Arabidopsis. Plant Physiol 2009, 150:84-95.

17. Frova C: The plant glutathione transferase gene family: genomic structure, functions, expression and evolution. Physiol Plant 2003, 119:469-479.

18. Higo K, Ugawa $Y$, Iwamoto M, Korenaga T: Plant cis-acting regulatory DNA elements (PLACE) database. Nucleic Acids Res 1999, 27:297-300.

19. Wang $C$, Gao CQ, Wang LQ, Yang CP, Zheng L, Wang YC: Comprehensive transcriptional profiling of $\mathrm{NaHCO}_{3}$-stressed Tamarix hispida roots reveals networks of responsive genes. Plant Mol Biol 2013. doi:10.1007/s11103013-0124-2. 
20. Jefferson RA: The GUS reporter gene system. Nature 1989, 342:837-838.

21. Jefferson RA, Kavanagh TA, Bevan MW: GUS fusions: betaglucuronidase as a sensitive and versatile gene fusion marker in higher plants. EMBO J 1987, 6:3901-3907.

22. Pfaffl MW, Horgan GW, Dempfle L: Relative expression software tool (REST) for group-wise comparison and statistical analysis of relative expression results in real-time PCR. Nucleic Acids Res 2002, 30:36.

23. Zhang $X$, Wang $L$, Meng $H$, Wen $H$, Fan $Y$, Zhao J: Maize ABP9 enhances tolerance to multiple stresses in transgenic Arabidopsis by modulating ABA signaling and cellular levels of reactive oxygen species. Plant Mol Biol 2011, 75:365-378.

24. Kim M, Ahn JW, Jin UH, Choi D, Paek KH, Pai HS: Activation of the programmed cell death pathway by inhibition of proteasome function in plants. J Biol Chem 2003, 278:19406-19415.

25. Pei Z-M, Murata Y, Benning G, Thomine S, Klusener B, Allen GJ, Grill E, Schroeder Jl: Calcium channels activated by hydrogen peroxide mediate abscisic acid signalling in guard cells. Nature 2000, 406:731-734.

26. Thordal-Christensen $\mathrm{H}$, Zhang Z, Wei $Y$, Collinge DB: Subcellular localization of $\mathrm{H}_{2} \mathrm{O}_{2}$ in plants. $\mathrm{H}_{2} \mathrm{O}_{2}$ accumulation in papillae and hypersensitive response during the barley-powdery mildew interaction. Plant J 1997, 11:1187-1194.

27. Terada T, Maeda H, Okamoto K, Nishinaka T, Mizoguchi T, Nishihara T: Modulation of glutathione S-transferase activity by a thiol/disulfide exchange reaction and involvement of thioltransferase. Arch Biochem Biophys 1993, 300:495-500.

doi:10.1186/1471-2229-13-151

Cite this article as: Ji et al: The bZIP protein from Tamarix hispida,

ThbZIP1, is ACGT elements binding factor that enhances abiotic stress signaling in transgenic Arabidopsis. BMC Plant Biology 2013 13:151.

\section{Submit your next manuscript to BioMed Central and take full advantage of:}

- Convenient online submission

- Thorough peer review

- No space constraints or color figure charges

- Immediate publication on acceptance

- Inclusion in PubMed, CAS, Scopus and Google Scholar

- Research which is freely available for redistribution 